\title{
The Prognostic Comparison Between Hepatocellular Carcinoma with Portal Vein Tumor Thrombus and Bile Duct Cancer Thrombus After Liver Resection
}

This article was published in the following Dove Press journal: Cancer Management and Research

\author{
Xin Yang $\mathbb{D}^{1, *}$ \\ Ying Zhu $\mathbb{D}^{1, *}$ \\ Xia Zhao ${ }^{2, *}$ \\ Jian-hua Li $\mathbb{D}^{1, *}$ \\ Da Xu' \\ Hu-Liang Jia' \\ Ju-bo Zhang ${ }^{3}$
}

'Department of General Surgery, Huashan Hospital \& Cancer Metastasis Institute, Fudan University, Shanghai, People's Republic of China; ${ }^{2}$ Health Management Center, Huashan Hospital, Fudan University, Shanghai, People's Republic of China; ${ }^{3}$ Department of Infectious Diseases, Huashan Hospital, Fudan University, Shanghai, People's Republic of China

*These authors contributed equally to this work
Correspondence: Ju-bo Zhang

Department of Infectious Diseases, Huashan Hospital, Fudan University, 12 Urumqi Road (M), Shanghai 200040,

People's Republic of China

Tel +86-2I-52887/70

Email drzhangjubo@I63.com

Hu-Liang Jia

Department of General Surgery, Huashan Hospital, Cancer Metastasis Institute,

Fudan University, 12 Urumqi Road (M),

Shanghai 200040, People's Republic of

China

Tel +86-2I-52887I75

Email jbl-I@।63.com
Background and Objective: Hepatocellular carcinoma (HCC) often invades the portal vein and its branches to form portal vein tumor thrombus (PVTT), and it rarely spreads into the bile ducts to cause bile duct tumor thrombus (BDTT). However, the clinical prognosis of patients with the two types of tumor thrombus is different. In this manuscript, we plan to compare the prognosis of HCC with PVTT and BDTT for further clinical treatment.

Patients and Methods: A total of 60 patients including $48 \mathrm{HCC}$ cases with PVTT and 12 HCC cases with BDTT were enrolled in the study. The medical records were collected from participants. The follow-up was performed in 3 years post-hepatectomy. Statistical analysis was performed to explore the relationship between tumor thrombus with clinicopathological characteristics, to determine the significant preoperative factors influencing overall survival (OS) and time to recurrence (TTR), and to establish the survival and recurrent curves.

Results: HCC with BDTT or PVTT often combined with viral hepatitis B, accompanied by varying degrees of cirrhosis, and high AFP level (68.3\%), complete tumor capsule (76.7\%), and larger tumor size $(85.0 \%)$. Furtherly, patients with HCC and BDTT tended to have higher total bilirubin (TB) and more possibility of lymph node metastases. The multivariate Cox hazard analyses also revealed that both tumor size and tumor thrombus could be taken as independent prognostic indicators of HCC patients. Survival curves showed that the 1-, 2and 3-year OS or DFS rates of HCC patients with BDTT were significantly lower than those of HCC patients with PVTT, respectively.

Conclusion: Tumor thrombus is an independent risk factor for poor survival and high recurrence in HCC. HCC patients with BDTT had shorter overall survival and higher tumor recurrence rate compared to HCC patients with PVTT.

Keywords: hepatocellular carcinoma, portal vein tumor thrombus, bile duct cancer thrombus, prognosis

\section{Synopsis}

Our study observes the prognosis of HCC with portal vein tumor thrombus (PVTT) and $\mathrm{HCC}$ with bile duct tumor thrombus (BDTT) to provide a reference for further clinical treatment. Our observations suggest that liver cancer with PVTT or BDTT is an independent risk factor for poor survival and high recurrence in HCC. HCC patients with BDTT had shorter overall survival and higher tumor recurrence rate compared to HCC patients with PVTT. It should be diagnosed and treated as early and precise as possible to improve the prognosis of the patients. 


\section{Introduction}

Hepatocellular carcinoma, as an extremely malignant tumor, is the second most lethal malignancy worldwide, and responsible for more than 700,000 deaths annually. ${ }^{1,2}$ Owing to the prevalence of hepatitis $\mathrm{B}$ and $\mathrm{C}$, the incidence rate of liver cancer is higher in Asia. ${ }^{2}$ Unlike other digestive system tumors that are prone to invade lymph nodes, HCC is considered as its characteristic of a strong propensity to invade the surrounding liver vasculature. ${ }^{2-4}$ The portal vein and its branches, as a most common invasion place of $\mathrm{HCC}$, are found to be invaded in about $31.4 \% \sim 34 \%$ of all HCC patients according to clinical data. ${ }^{4}$ According to the European Association for the Study of the Liver/the American Association for the Study of the Liver Disease/Barcelona Clinic for Liver Cancer (EASL/AASLD/ BCLC) Staging System and treatment guidelines, the portal vein invasion, or portal vein tumor thrombosis (PVTT), is regarded as being indicative of an advanced stage of the disease with almost little hope to be cured. ${ }^{5-7}$ The only proposed treatment option for this group of patients is moleculartargeted therapy drug including sorafenib and lenvatinib. ${ }^{7,8}$ However, the reported median survival time (MST) of patients with advanced HCC treated with sorafenib or lenvatinib is as short as 9.8 or 11.5 months. ${ }^{9}$ Conversely, more aggressive anticancer treatments are recommended for selected patients with HCC and PVTT by Chinese, Japanese, South Korean, and Asia-Pacific clinical practice guidelines. ${ }^{10-13}$ The surgical resection is recommended by the Chinese expert consensus for some patients with HCC and PVTT limited to a first-order branch of the main portal vein or above. ${ }^{10}$ Meanwhile, some promising survival outcomes being reported using surgical resection and other modalities including molecular target therapy, radiotherapy, and transcatheter arterial chemoembolization (TACE). ${ }^{10,14,15}$ Recently, as more aggressive therapeutic schemes developing, such as operation, interventional radiotherapy including radio-embolization, molecularly targeted therapy and other multidisciplinary treatments, the life quality is greatly improved and the survival time is prolonged gradually in some patients, ${ }^{14,15}$ even some of which were used as the downgrade treatment for patients without the possibilities of surgery. Recent advances in surgical techniques and perioperative management have promoted liver resection to become a reasonably safe treatment option with an acceptable mortality and morbidity rate. ${ }^{16-18}$ At present, the aggressive surgical resection for HCC with PVTT has been proposed in several centers. ${ }^{17,18}$

However, different from PVTT, HCC with bile duct tumor thrombus (BDTT) is a relatively rare event in clinical practice which accounts for only $1.2 \pm 12.9 \%$ of total HCC cases. ${ }^{19}$ As a result of a lack of the right awareness of HCC with BDTT, some patients fail to receive effective treatment. Presently, the diagnosis of BDTT mainly depends on clinical symptoms, physical examinations, image studies, surgical exploration and postoperative pathological results. According to the type of BDTT, patients with HCC and BDTT may undergo radical resection, thrombectomy through a choledochotomy, palliative internal and external bile duct drainage including, or transarterial chemoembolization (TACE). Depending on the patient's condition, sometimes routine percutaneous transhepatic cholangial drainage (PTCD) or endoscopic nasobiliary drainage (ENBD) was performed before surgery to reduce the degree of jaundice. The active surgical resection may benefit some selected patients. ${ }^{20,21}$

When patients with $\mathrm{HCC}$ have developed into portal vein tumor thrombus (PVTT) or bile duct tumor thrombus (BDTT), it was always judged that the condition has stepped into an advanced stage, furtherly followed by intrahepatic and extrahepatic metastases, portal hypertension, jaundice and ascites in a short period. If not given treatment in time, the median survival time of patients will be less than 6 months. Therefore, PVTT and BDTT are recognized as an important prognosis factor for patients with HCC. As the diagnosis imaging is developed and more awareness is improved regarding the recognition of these types of disease, the correct preoperative diagnosis and the prompt treatment are given. Due to the different biological characteristics of bile duct and portal vein tumor thrombus, the prognosis of PVTT and BDTT after surgery is different. In this article, we clarified the long-term surgical outcomes of HCC with the two types of tumor thrombus.

\section{Patients and Methods}

\section{Patients}

A total of 60 patients including $48 \mathrm{HCC}$ cases with PVTT and $12 \mathrm{HCC}$ cases with BDTT were enrolled in the study. Patients received curative resection for HCC at the department of General surgery, Huashan Hospital, Fudan University (Shanghai, China) between January 2014 and December 2016. All the patients meet the following inclusion criteria (a) a definite clinically diagnosed with HCC and postoperative pathological diagnosis of HCC, (b) R0 resection of all patients based on histologic examinations. The tumor thrombus is removed during surgery. (c) The TNM staging is in accordance with the AJCC/UICC 2017 TNM staging standard. (d) Classification of liver function 
was graded according to Child-Pugh classification criteria. (e) Without distant metastasis, (f) patients did not receive any treatment before surgery. (g) The diagnosis of portal vein tumor thrombus was based on pathological tissue slices, preoperative imaging and confirmed in the surgery. (h) Patients were generally recommended to undergo trans-arterial chemoembolization (TACE) therapy at 1 month after surgery. All cases had complete laboratory values, imaging results, clinical and pathology data, and treatment information, including gender, age, $\mathrm{HBsAg}$, cirrhosis, preoperative AFP, ALT, and tumor size, tumor number, tumor capsule, the degree of differentiation, distant metastasis, PVTT and BDTT stage, TNM staging. Clinical samples were collected from patients after obtaining informed consent in accordance with a protocol approved by the Ethics Committee of Huashan Hospital (Shanghai, China), which was in accordance with the Declaration of Helsinki.

\section{The Classification of PVTT and BDTT}

It is greatly important to select the appropriate therapeutic strategies depending on the classification of PVTT. The portal vein invasion classification $(\mathrm{Vp})$ has been used worldwide since it was introduced in 1987 by the Liver Cancer Study Group of Japan. ${ }^{22,23}$ The Eastern Hepatobiliary classification (also known as Cheng's classification) was devised in 2007. ${ }^{24}$ The Details of the Vp classification and Cheng's classification are provided in the Supplementary Table S1. No such classification system has been developed yet in the west. Hepatocellular carcinoma with BDTT was classified according to the location of BDTT, as proposed by $\mathrm{Ueda}^{25}$ (Supplementary Table S1). The patients with PVTT selected in this study are at the stage of Vp1-3 (Cheng's classification type I or II), while those with BDTT were classified as Ueda type I or II.

\section{Transcatheter Arterial Chemoembolization (TACE)}

HCC patients with PVTT or BDTT were generally recommended to undergo TACE at 1 month after surgery. Whether the patients followed the physician's recommendation mainly depended on their socioeconomic status and compliance with the doctors. TACE was conducted with a conventional procedure. In brief, hepatic arteriography and superior mesenteric arterial portovenography were performed first to verify whether tumor staining occured, especially for the operative area. The arteries supplying the tumors were catheterized superselectively as close as possible to the suspicious tumor, followed by infusion of 1 $\mathrm{g}$ of 5 -fluorouracil, $150 \mathrm{mg}$ of oxaliplatin, and $30 \mathrm{mg}$ pirarubicin mixed with $5-10 \mathrm{~mL}$ of lipiodol. The dosage of chemotherapeutic agents or lipiodol was adjusted according to the body weight and liver function.

\section{Statistical Analysis}

The statistical analysis was performed with IBM SPSS Statistics 20 statistical software. The measurement data of normal distribution are expressed by "mean \pm standard deviation", and Student's $t$-test was used to analyze quantitative variables between two groups. Fisher's exact test was used to analyze categorical data. Survival curves were established using the Kaplan-Meier method and compared using the Logrank test. $P<0.05$ in the results were statistically different and $P<0.01$ was statistically significantly different by a two-tailed test.

\section{Results}

\section{The Diagnosis and Treatment of HCC with PVTT or BDTT}

Patients with liver cancer and tumor thrombus were preliminarily diagnosed by imaging including B-mode ultrasound, enhanced CT, and enhanced magnetic resonance imaging. All tumor thrombi were confined to the first branch or higher grade of the portal vein or intrahepatic bile duct did not invade or extend to the main portal vein or common hepatic duct (Figure 1A and B). After a comprehensive and detailed evaluation before the surgery, a reasonable surgical plan is made. Based on the systemic and tumor conditions, we perform liver resections on patients, including regular anatomical hepatic resection of the lobes or the segments, or local irregular liver resections, and tumor thrombus are removed as a whole during surgery. In the present study, 30 patients with PVTT and 7 patients with BDTT were treated by the anatomical hepatectomy, while 18 patients with PVTT and 5 patients with BDTT were treated by non-anatomical hepatectomy. The detailed descriptions are shown in Supplementary Table S2. The tumor and tumor thrombus tissue were almost visually observed after surgery (Figure 1A and B). The final diagnosis depends on the current pathological diagnosis of the microscope. 
A
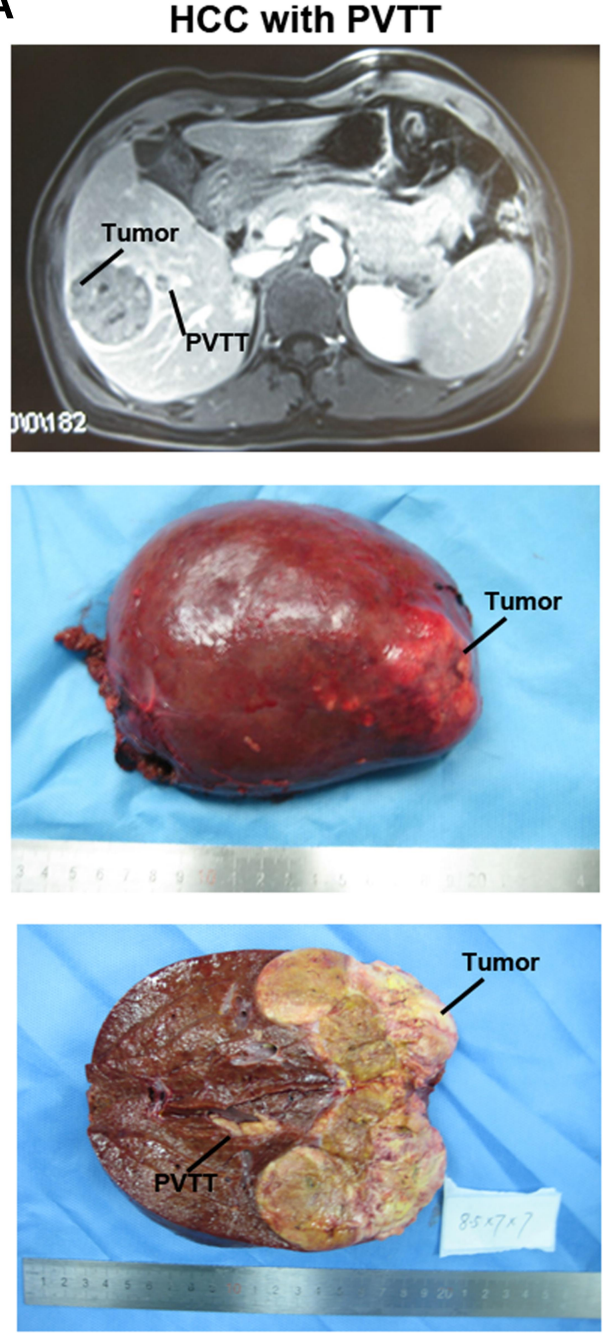

B
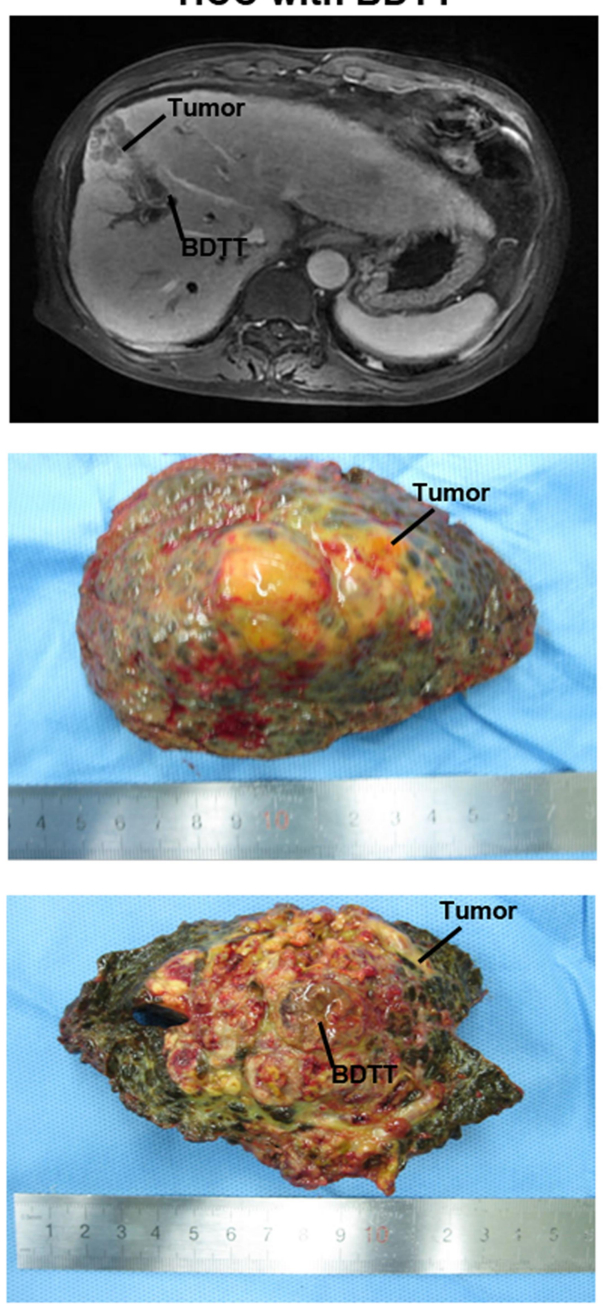

Figure I The Location of hepatocellular carcinoma with (A) portal vein tumor thrombus and (B) bile duct cancer thrombus in magnetic resonance imaging, naked eye specimens, and specimen sections. All tumor thrombi were confined to the first branch or higher grade of the portal vein or intrahepatic bile duct, did not invade or extend to the main portal vein or common hepatic duct. The Location of hepatocellular carcinoma and tumor thrombus in (upper) magnetic resonance imaging, (middle) naked eye specimens, and (lower) specimen sections.

\section{The Clinicopathological Characteristics in HCC with PVTT or BDTT}

The characteristics of a total of 60 patients are included in Table 1. Male and the age more than 50 years old accounted for $78.3 \%$ and $83.3 \%$ of the study population, respectively. Almost all individuals (96.7\%) enrolled in our research have a background of hepatitis B. At the same time, the proportion of liver cirrhosis is as high as $75 \%$. Among the 60 patients, 48 HCC patients ( $80 \%$ ) were accompanied by the vascular invasion of portal vein, and 12 HCC patients $(20 \%)$ were diagnosed as HCC with bile duct tumor thrombus. Mostly, the patients with PVTT $(80 \%)$ or BDTT $(20 \%)$ had the feathers with high AFP level more than $20 \mathrm{ng} / \mathrm{mL}$ (68.3\%), low CA199 level less than $37 \mathrm{Ku} / \mathrm{L}(76.7 \%)$, complete tumor capsule $(76.7 \%)$, and tumor size more than $5 \mathrm{~cm}(85.0 \%)$. The less lymph node metastasis $(90.0 \%)$ occurs to the enrolled population which is mostly diagnosed at a stage TNM III/IV (86.7\%).

\section{The Association of Tumor Thrombus with Clinicopathological Characteristics}

Next, we evaluated the association between tumor thrombosis and clinicopathological characteristics of $\mathrm{HCC}$ patients. The correlative analysis revealed that tumor thrombus was significantly correlated with total bilirubin $(P<0.001)$ or lymph node metastasis $(P=0.012)$ (Table 2$)$, respectively. Further analysis found that patients with liver cancer and bile duct cancer thrombus tended to have 
Table I Clinicopathological Characteristics of HCC Patients $(n=60)$

\begin{tabular}{|c|c|c|}
\hline Characteristics & Case Number & $\%$ \\
\hline \multicolumn{3}{|l|}{ Gender } \\
\hline Female & 13 & 21.7 \\
\hline Male & 47 & 78.3 \\
\hline \multicolumn{3}{|l|}{ Age (years) } \\
\hline$<50$ & 10 & 16.7 \\
\hline$\geq 50$ & 50 & 83.3 \\
\hline \multicolumn{3}{|l|}{$\mathrm{HBsAg}$} \\
\hline Negative & 2 & 3.3 \\
\hline Positive & 58 & 96.7 \\
\hline \multicolumn{3}{|l|}{ Cirrhosis } \\
\hline No & 15 & 25.0 \\
\hline Yes & 45 & 75.0 \\
\hline \multicolumn{3}{|l|}{ ALT (U/L) } \\
\hline$<75$ & 49 & 81.7 \\
\hline$\geq 75$ & II & 18.3 \\
\hline \multicolumn{3}{|l|}{ TB $(\mu \mathrm{mol} / \mathrm{L})$} \\
\hline$\leq 200$ & 55 & 91.7 \\
\hline$>200$ & 5 & 8.3 \\
\hline \multicolumn{3}{|l|}{ ALB $(g / L)$} \\
\hline$\geq 35$ & 25 & 41.7 \\
\hline$<35$ & 35 & 58.3 \\
\hline \multicolumn{3}{|l|}{$\operatorname{AFP}(\mathrm{ng} / \mathrm{mL})$} \\
\hline$<20$ & 19 & 31.7 \\
\hline$\geq 20$ & $4 I$ & 68.3 \\
\hline \multicolumn{3}{|l|}{ CAI99 (kU/L) } \\
\hline$<37$ & 46 & 76.7 \\
\hline$\geq 37$ & 14 & 23.3 \\
\hline \multicolumn{3}{|l|}{ Tumor size $(\mathrm{cm})$} \\
\hline$<5$ & 9 & 15.0 \\
\hline$\geq 5$ & 51 & 85.0 \\
\hline \multicolumn{3}{|l|}{ Tumor number } \\
\hline Single & 44 & 53.5 \\
\hline Multiple & 16 & 46.5 \\
\hline \multicolumn{3}{|l|}{ Tumor capsule } \\
\hline Complete & 46 & 76.7 \\
\hline None & 14 & 23.3 \\
\hline \multicolumn{3}{|l|}{ tumor thrombosis } \\
\hline PVTT & 48 & 80.0 \\
\hline BDTT & 12 & 20.0 \\
\hline \multicolumn{3}{|c|}{ Lymph node metastasis } \\
\hline No & 54 & 90.0 \\
\hline Yes & 6 & 10.0 \\
\hline
\end{tabular}

(Continued)
Table I (Continued).

\begin{tabular}{|l|l|l|}
\hline Characteristics & Case Number & $\%$ \\
\hline Tumor differentiation & & \\
I/II & 23 & 38.3 \\
III/IV & 37 & 61.7 \\
\hline TNM stage & & \\
I/II & 8 & 13.3 \\
III/IV & 52 & 86.7 \\
\hline
\end{tabular}

Abbreviations: $\mathrm{HBsAg}$, hepatitis $B$ surface antigen; $A L T$, alanine transaminase; $T B$, total bilirubin; ALB, albumin; AFP, alpha-fetoprotein; CA199, Cancer antigen 199; PVTT, portal vein tumor thrombus; BDTT, bile duct tumor thrombus; AFP, alphafetoprotein; TNM, tumor-node-metastasis staging system. Tumor differentiation was evaluated by Edmondson grade.

higher total bilirubin (TB) and more possibility of lymph node metastases. However, the two features do not exist in patients with liver cancer and portal vein tumor thrombus. There was no significant correlation between tumor thrombosis and the remaining clinical characteristics including gender, age, HBsAg (hepatitis B surface antigen), Cirrhosis, alanine transaminase (ALT), albumin (ALB), serum AFP level, serum CA199 level, tumor size, tumor number, tumor capsule, tumor differentiation, TNM stage (Table 2).

\section{The Prognostic Value of Tumor Thrombus in HCC Patients}

Furthermore, we performed the prognostic analysis in hepatocellular carcinoma with portal vein tumor thrombus and bile duct cancer thrombus after liver resection. Univariate analysis showed that tumor size and tumor thrombus were significantly associated with overall survival (OS) ( $P=0.038$ and $P=0.011$, respectively) and time to recurrence (TTR) $(P=0.018$ and $P=0.005$, respectively) (Table 3). However, the other enrolled pathological features showed no association with OS or TTR. The multivariate Cox hazard analyses also revealed that both tumor size and tumor thrombus could be taken as independent prognostic indicators of HCC patients (OS: $P=0.049$ and $P=0.023$, respectively; and TTR: $P=0.030$ and $P=0.010$, respectively) (Table 3). Collectively, the results demonstrated that tumor size and tumor thrombus is an independent prognostic indicator and associated with shorter OS and TTR in HCC patients.

Kaplan-Meier survival curves showed that the 1-, 2- and 3-year overall survival (OS) rates of HCC patients with 
Table 2 The Association of Tumor Thrombosis with Clinicopathological Characteristics of HCC Patients

\begin{tabular}{|c|c|c|c|}
\hline \multirow[t]{2}{*}{ Variable } & \multicolumn{2}{|c|}{ Tumor Thrombosis $(n=60)$} & \multirow[t]{2}{*}{$P$ value } \\
\hline & $\begin{array}{l}\text { BDTT } \\
(n=\mid 2)\end{array}$ & $\begin{array}{l}\text { PVTT } \\
(n=48)\end{array}$ & \\
\hline \multicolumn{4}{|l|}{ Gender } \\
\hline Female & $4(33.3 \%)$ & $9(18.8 \%)$ & 0.234 \\
\hline Male & $8(66.7 \%)$ & $39(81.2 \%)$ & \\
\hline \multicolumn{4}{|l|}{ Age (years) } \\
\hline$<50$ & $\mathrm{I}(8.3 \%)$ & $9(18.8 \%)$ & 0.354 \\
\hline$\geq 50$ & II(91.7\%) & $39(81.2 \%)$ & \\
\hline \multicolumn{4}{|l|}{$\mathrm{HBsAg}$} \\
\hline Positive & $12(100.0 \%)$ & $46(95.8 \%)$ & 0.637 \\
\hline Negative & $0(0.0 \%)$ & $2(4.2 \%)$ & \\
\hline \multicolumn{4}{|l|}{ Cirrhosis } \\
\hline Yes & $10(83.3 \%)$ & $35(72.9 \%)$ & 0.369 \\
\hline No & $2(16.7 \%)$ & $13(27.1 \%)$ & \\
\hline \multicolumn{4}{|l|}{ ALT (U/L) } \\
\hline$<75$ & $10(83.3 \%)$ & $39(81.2 \%)$ & 0.618 \\
\hline$\geq 75$ & $2(16.7 \%)$ & $9(18.8 \%)$ & \\
\hline \multicolumn{4}{|l|}{ TB $(\mu \mathrm{mol} / \mathrm{L})$} \\
\hline$\leq 200$ & $7(58.3 \%)$ & $48(100.0 \%)$ & 0.000 \\
\hline$>200$ & $5(41.7 \%)$ & $0(0.0 \%)$ & \\
\hline \multicolumn{4}{|l|}{ ALB (g/L) } \\
\hline$\geq 35$ & $7(58.3 \%)$ & $18(37.5 \%)$ & 0.163 \\
\hline$<35$ & $5(41.7 \%)$ & $30(62.5 \%)$ & \\
\hline \multicolumn{4}{|l|}{ AFP (ng/mL) } \\
\hline$<20$ & $5(41.7 \%)$ & $14(29.2 \%)$ & 0.307 \\
\hline$\geq 20$ & $7(58.3 \%)$ & $34(70.8 \%)$ & \\
\hline \multicolumn{4}{|l|}{ CA199 (kU/L) } \\
\hline$<37$ & $10(83.3 \%)$ & $36(75.0 \%)$ & 0.426 \\
\hline$\geq 37$ & $2(16.7 \%)$ & $12(25.0 \%)$ & \\
\hline \multicolumn{4}{|l|}{ Tumor size $(\mathrm{cm})$} \\
\hline$<5$ & $3(25.0 \%)$ & $6(12.5 \%)$ & 0.191 \\
\hline$\geq 5$ & $9(75.0 \%)$ & $42(87.5 \%)$ & \\
\hline \multicolumn{4}{|l|}{ Tumor number } \\
\hline Single & II(9I.7\%) & $33(68.8 \%)$ & 0.103 \\
\hline Multiple & $\mathrm{I}(8.3 \%)$ & $15(31.2 \%)$ & \\
\hline \multicolumn{4}{|l|}{ Tumor capsule } \\
\hline None & $\mathrm{I}(8.3 \%)$ & $13(27.1 \%)$ & 0.161 \\
\hline Complete & II(9I.7\%) & $35(72.9 \%)$ & \\
\hline \multicolumn{4}{|l|}{$\begin{array}{l}\text { Lymph node } \\
\text { metastasis }\end{array}$} \\
\hline No & $8(66.6 \%)$ & $46(95.8 \%)$ & 0.012 \\
\hline Yes & $4(33.3 \%)$ & $2(4.2 \%)$ & \\
\hline
\end{tabular}

(Continued)
Table 2 (Continued)

\begin{tabular}{|c|l|l|l|}
\hline \multirow{2}{*}{ Variable } & \multicolumn{2}{|l|}{ Tumor Thrombosis (n=60) } & \multirow{2}{*}{ P value } \\
\cline { 2 - 3 } & $\begin{array}{l}\text { BDTT } \\
(\mathbf{n = 1 2 )}\end{array}$ & $\begin{array}{l}\text { PVTT } \\
(\mathbf{n = 4 8 )}\end{array}$ & \\
\hline $\begin{array}{c}\text { Tumor differentiation } \\
\text { I/II }\end{array}$ & $6(50.0 \%)$ & $17(35.4 \%)$ & \multirow{2}{*}{0.272} \\
III/IV & $6(50.0 \%)$ & $31(64.6 \%)$ & \\
\hline $\begin{array}{c}\text { TNM stage } \\
<5\end{array}$ & $2(16.7 \%)$ & $6(12.5 \%)$ & \multirow{2}{*}{0.507} \\
$\geq 5$ & $10(83.3 \%)$ & $42(87.5 \%)$ & \\
\hline
\end{tabular}

Notes: Analysis was conducted using $\chi^{2}$ tests and Fisher's exact tests. Bold indicates significant values.

Abbreviations: $\mathrm{HBs} A$, hepatitis $B$ surface antigen; $A L T$, alanine transaminase; TB, total bilirubin; ALB, albumin; AFP, alpha-fetoprotein; CAI99, Cancer antigen 199; PVTT, portal vein tumor thrombus; BDTT, bile duct tumor thrombus; AFP, alphafetoprotein; TNM, tumor-node-metastasis staging system. Tumor differentiation was evaluated by Edmondson grade.

BDTT $(41.7 \%, 33.3 \%$ and $33.3 \%$, respectively) were significantly lower than HCC patients with PVTT (57.6\%, $54.6 \%$ and $54.6 \%$, respectively) ( $P=0.0212$, Figure $2 \mathrm{~A})$. In addition, the 1-, 2- and 3-year tumor recurrence rates of HCC patients with BDTT $(51.3 \% .80 .5 \%$ and $90.3 \%$, respectively) were significantly higher than HCC with PVTT group (36.6\%, $52.3 \%$ and $72.4 \%$, respectively) $(P=0.0246$, Figure 2B). The results showed that HCC patients with BDTT had shorter overall survival (OS) and higher tumor recurrence rate compared to HCC patients with PVTT.

\section{Discussion}

Hepatocellular carcinoma is one of the most fatal malignancies worldwide. Owing to the biological characteristics of liver cancer and the anatomical characteristics of the liver, it is prone to invade intrahepatic vessels, especially the portal venous system, rarely in the bile duct system.

Once PVTT is developed, and then it progressed rapidly to cause portal hypertension, hepatocellular jaundice, and intractable ascites, which seriously affect the prognosis of patients. Therefore, according to the situation, appropriate interventions are given to these patients. However, it is of great importance to decide the appropriate therapeutic strategies by the classification of PVTT. At present, the Vp classification and Cheng's classification of PVTT have been introduced and used worldwide. ${ }^{22-24}$ The treatment strategies for patients who have HCC with PVTT differ substantially between the west (Europe and America) and the east (Asia-Pacific region). According to 
Table 3 Univariate and Multivariate Analyses of Factors Associated with OS and TTR of HCC Patients $(n=60)$

\begin{tabular}{|c|c|c|c|c|}
\hline \multirow[t]{2}{*}{ Clinical Variables } & \multicolumn{2}{|c|}{ Overall Survival } & \multicolumn{2}{|c|}{ Time To Recurrence } \\
\hline & HR (95\% Cl) & $P$ value & HR (95\% Cl) & P value \\
\hline \multicolumn{5}{|l|}{ Univariate analysis } \\
\hline Gender (female versus male) & $0.93(0.16-6.35)$ & 0.126 & $1.62(0.52-4.23)$ & 0.312 \\
\hline Age $(\geq 50$ versus $<50$ years $)$ & $0.72(0.37-1.92)$ & 0.592 & $1.06(0.35-2.45)$ & 0.913 \\
\hline $\mathrm{HBsAg(positive} \mathrm{verse} \mathrm{negative)}$ & $1.28(0.73-4.12)$ & 0.985 & $3.03(0.84-6.32)$ & 0.518 \\
\hline Cirrhosis (yes versus no) & $0.75(0.32-2.20)$ & 0.649 & $0.81(0.39-2.52)$ & 0.754 \\
\hline ALT ( $\geq 75$ versus $<75 \mathrm{U} / \mathrm{L})$ & $1.03(0.93-3.56)$ & 0.802 & $1.29(0.85-3.11)$ & 0.843 \\
\hline $\mathrm{TB}(\leq 200$ versus $>200 \mu \mathrm{mol} / \mathrm{L})$ & $0.63(0.35-1.57)$ & 0.348 & $0.98(0.36-2.69)$ & 0.711 \\
\hline$A L B(\geq 35$ versus $<35 g / L)$ & $0.87(0.32-1.85)$ & 0.707 & $0.6 \mathrm{I}(0.23-1.54)$ & 0.602 \\
\hline AFP ( $\geq 20$ versus $<20 \mathrm{ng} / \mathrm{mL}$ ) & $1.62(0.74-2.35)$ & 0.816 & $0.79(0.66-1.62)$ & 0.412 \\
\hline CAI99 ( $\geq 37$ versus<37kU/L) & $0.55(0.29-1.07)$ & 0.078 & $0.96(0.65-1.56)$ & 0.681 \\
\hline Tumor size $(\geq 5 \mathrm{~cm}$ versus $<5 \mathrm{~cm})$ & $3.00(1.06-8.4 I)$ & 0.038 & $3.32(1.42-9.21)$ & 0.018 \\
\hline Tumor number (multiple versus single) & $0.49(0.18-1.26)$ & 0.131 & $1.25(0.46-2.14)$ & 0.263 \\
\hline Tumor capsule (complete versus none) & $0.77(0.20-1.83)$ & 0.467 & $0.82(0.47-2.26)$ & 0.614 \\
\hline Tumor thrombus (PVTT versus BDTT) & $4.61(1.44-9.26)$ & 0.011 & $3.43(1.29-9.67)$ & 0.005 \\
\hline Lymph node metastasis (yes versus no) & $0.98(0.35-6.57)$ & 0.936 & $2.31(0.27-9.49)$ & 0.442 \\
\hline Tumor differentiation (III/IV versus I-II) & $0.86(0.38-2.25)$ & 0.621 & $0.73(0.39-1.48)$ & 0.256 \\
\hline TNM stage (III/IV versus I/II) & $0.92(0.17-4.39)$ & 0.865 & $0.56(0.22-1.46)$ & 0.225 \\
\hline \multicolumn{5}{|l|}{ Multivariate analysis } \\
\hline Tumor size $(\geq 5 \mathrm{~cm}$ versus $<5 \mathrm{~cm})$ & $2.86(0.92-8.36)$ & 0.049 & $3.15(1.28-9.02)$ & 0.030 \\
\hline Tumor thrombus (PVTT versus BDTT) & $3.7 \mathrm{I}(1.24-8.47)$ & 0.023 & $2.93(1.11-7.89)$ & 0.010 \\
\hline
\end{tabular}

Notes: Analysis was conducted using univariate analysis or multivariate Cox proportional hazards regression. Bold indicates significant values.

Abbreviations: $\mathrm{HBsAg}$, hepatitis B surface antigen; ALT, alanine transaminase; TB, total bilirubin; ALB, albumin; AFP, alpha-fetoprotein; CAI99, Cancer antigen I99;PVTT, portal vein tumor thrombus;BDTT, bile duct tumor thrombus; AFP, alpha-fetoprotein; TNM, tumor-node-metastasis staging system; Cl, confidence interval. Tumor differentiation was evaluated by Edmondson grade.

western guidelines, the molecular-targeted treatment is recommended for HCC patients with PVTT. Sorafenib was the first molecularly targeted agent to be approved for first-line treatment for patients with advanced HCC by the US Food and Drug Administration (FDA) and the
European Medicines Agency (EMA) in 2007, and lenvatinib was approved in 2018. ${ }^{7,8}$ In addition, regorafenib, ${ }^{26}$ cabozantinib $^{27}$ and ramucirumab ${ }^{28}$ are approved by the FDA and EMA for the second-line treatment of patients with advanced HCC, especially after sorafenib and
A

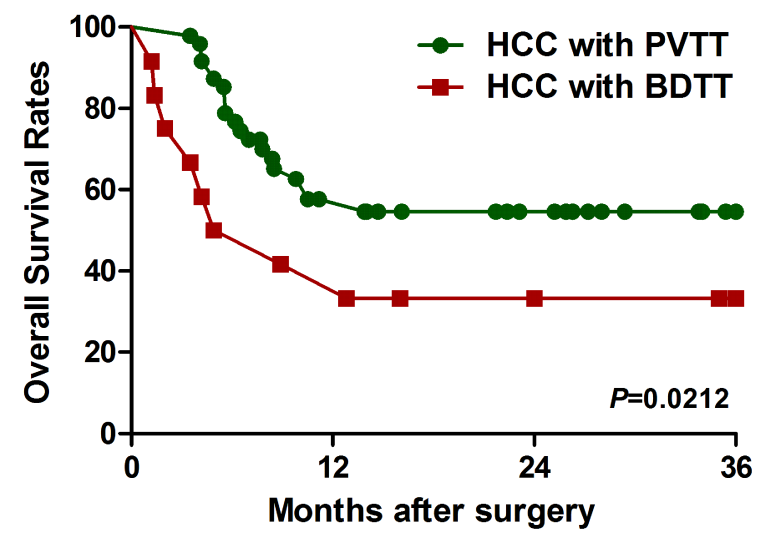

B

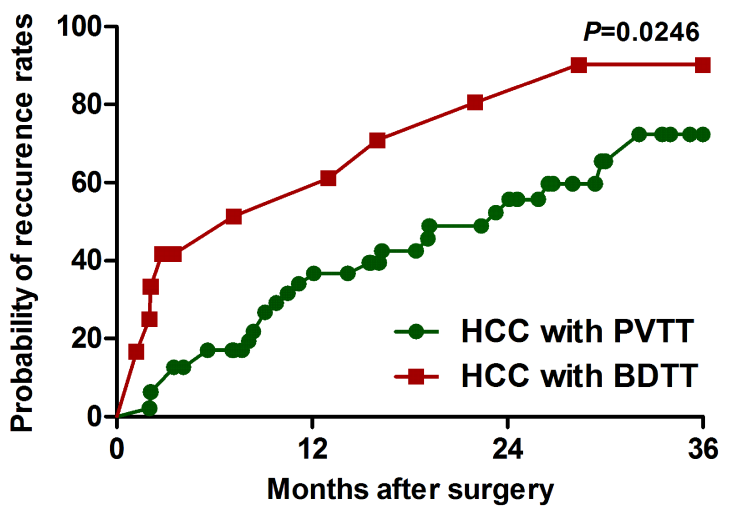

Figure 2 The prognostic comparison between hepatocellular carcinoma with portal vein tumor thrombus and bile duct cancer thrombus after liver resection by the analysis of Kaplan-Meier curves. Kaplan-Meier curves showed that HCC patients with bile duct cancer thrombus had (A) shorter overall survival and (B) higher tumor recurrence rate compared to HCC patients with portal vein tumor thrombus. P values were calculated by Log-rank test. 
lenvatinib failure. Conversely, more aggressive anticancer treatments are recommended for selected patients with HCC by Chinese, Japanese, South Korean, and AsiaPacific clinical practice guidelines. Surgical resection for some patients with HCC and PVTT limited to a first-order branch of the main portal vein or above were recommended in Chinese expert consensus. Using radiotherapy, transcatheter arterial chemoembolization (TACE), and other modalities were also reported to have promising survival outcomes. ${ }^{10,29,30}$

HCC with bile duct tumor thrombus (BDTT) is one of the rare and special types of liver cancer which was often misdiagnosed as biliary calculi, cholangiocarcinoma, liver failure, or advanced HCC for similar clinical presentations and imaging characteristics. ${ }^{31}$ Currently, it is believed that the occurrence of liver cancer combined with BDTT is related to the biological characteristics of the tumor, the body's microenvironment, the human immune status, the adjacent relationship between liver cancer and bile ducts, but not to the size of liver cancer. ${ }^{32,33}$ Tumor cells first invade the sub-epithelium of the adjacent small bile duct, and then they grow continuously along the bile duct wall to the extrahepatic bile duct. ${ }^{32,33}$ The presence of BDTT usually implies a poor prognosis. Due to the misunderstandings of HCC with BDTT that these patients are already in an advanced stage, some patients fail to receive more effective treatment. In fact, as to the patients with liver cancer combined with BDTT, early diagnosis and early active surgical intervention can make them obtain satisfactory survival results. $^{34,35}$ At present, the diagnosis of liver cancer combined with BDTT mainly depends on imaging examination including enhanced CT, MRCP and ERCP, etc., combined with clinical manifestations and intraoperative exploration, postoperative pathological examination. The commonly used Ueda classification combines surgical needs and facilitates prognosis judgment. $^{25}$ The simplified clinical typing is proposed by the Japanese scholar Satoh in 2000, which is suitable for clinical preliminary judgment of the location and prognosis of BDTT. ${ }^{36}$ BDTT may undergo radical resection, thrombectomy through a choledochotomy, palliative internal and external bile duct drainage, or trans-arterial chemoembolization (TACE) depending on the type of BDTT. ${ }^{21,37}$ However, patients with $\mathrm{HCC}$ and BDTT have a relatively high risk of early recurrence, resulting in poor prognosis even after curative hepatic resection or liver transplantation. $^{21}$ Therefore, postoperative preventive treatment is useful to improve the OS rate after the operation. Some observations have shown that tumor thrombectomy through a choledochotomy is contraindicated for surgical treatment of $\mathrm{HCC}$ with BDTT, as this procedure might cause tumor recurrence at the site of the choledochotomy probably due to intraoperative iatrogenic implantation. ${ }^{38}$ In conclusion, patients with HCC and BDTT have a relatively high rate of early recurrence after surgery. The prognosis of this type of HCC is extremely dismal, but the survival can be improved if complete tumor with BDTT clearance is achieved.

In this study, we retrospectively studied the clinicopathological features of 60 patients with HCC and PVTT or BDTT. After a comprehensive and detailed evaluation before surgery, a reasonable surgical plan was performed. All tumor thrombi were confined to the portal vein or intrahepatic bile duct of primary or higher grade and did not invade or extend to the main portal vein or common hepatic duct. We found that the clinical features of hepatocellular carcinoma with BDTT or PVTT are often combined with viral hepatitis $\mathrm{B}$, accompanied by varying degrees of cirrhosis. Further analysis found that patients with liver cancer with bile duct cancer thrombus tended to have higher total bilirubin and more possibility of lymph node metastases. Tumor thrombus is an independent prognostic indicator and associated with shorter OS and TTR in HCC patients. The results furtherly showed that HCC patients with BDTT had shorter overall survival and higher tumor recurrence rate compared to HCC patients with PVTT. However, more studies with a larger number of patients of HCC with BDTT or PVTT are needed to draw the final conclusion.

In summary, the liver cancer with PVTT or BDTT is an independent risk factor for poor survival and high recurrence in HCC. HCC patients with BDTT had shorter overall survival and higher tumor recurrence rate compared to HCC patients with PVTT. It should be diagnosed and treated as early and precise as possible to improve the prognosis of the patients.

\section{Acknowledgments}

This work was generously sponsored by the National Natural Science Foundation of China (81672365, 81672848 ) and China National Key Projects for Infectious Disease (2017ZX10203207).

\section{Disclosure}

The authors report no conflicts of interest for this work. 


\section{References}

1. Torre LA, Bray F, Siegel RL, Ferlay J, Lortet-Tieulent J, Jemal A. Global cancer statistics, 2012. CA Cancer J Clin. 2015;65:87-108. doi: $10.3322 /$ caac. 21262

2. Takeda H, Takai A, Inuzuka T, Marusawa H. Genetic basis of hepatitis virus-associated hepatocellular carcinoma: linkage between infection, inflammation, and tumorigenesis. $J$ Gastroenterol. 2017;52:26-38.

3. Park JW, Chen M, Colombo M, et al. Global patterns of hepatocellular carcinoma management from diagnosis to death: the BRIDGE study. Liver Int. 2015;35:2155-2166. doi:10.1111/liv.12818

4. Jian L, Zhang X-P, Zhong B-Y, et al. Management of patients with hepatocellular carcinoma and portal vein tumor thrombosis: comparing east and west. Lancet Gastroenterol Hepatol. 2019;4:721-730. doi:10.1016/S2468-1253(19)30178-5

5. European Association for the Study of the Liver. EASL Clinical Practice Guidelines: Management of hepatocellular carcinoma. Journal of Hepatology. 2018;69(1):182236. doi:10.1016/j.jhep.2018.03.019

6. Bruix J, Reig M, Sherman M. Evidence-based diagnosis, staging, and treatment of patients with hepatocellular carcinoma. Gastroenterology. 2016;150:835-853. doi:10.1053/j.gastro.2015.12.041

7. Marrero JA, Kulik LM, Sirlin CB, et al. Diagnosis, staging, and management of hepatocellular carcinoma: 2018 practice guidance by the American Association for the study of liver diseases. Hepatology. 2018;68:723-750. doi:10.1002/hep.29913

8. Llovet JM, Ricci S, Mazzaferro V, et al. Sorafenib in advanced hepatocellular carcinoma. $N$ Engl J Med. 2008;359:378-390. doi:10. 1056/NEJMoa0708857

9. Kudo M, Finn RS, Qin S, et al. Lenvatinib versus sorafenib in first-line treatment of patients with unresectable hepatocellular carcinoma: a randomised Phase 3 non-inferiority trial. Lancet. 2018;391:1163-1173. doi:10.1016/S0140-6736(18)30207-1

10. Cheng S, Yang J, Shen F, et al. Multidisciplinary management of hepatocellular carcinoma with portal vein tumor thrombus eastern hepatobiliary surgical hospital consensus statement. Oncotarget. 2016;7:40816-40829.

11. Kudo M, Kitano M, Sakurai T, Nishida N. General rules for the clinical and pathological study of primary liver cancer, nationwide follow-up survey and clinical practice guidelines: the outstanding achievements of the liver cancer study group of Japan. Dig Dis. 2015;33:765-770. doi:10.1159/000439101

12. Korean Liver Cancer Study Group, National Cancer Center, Korea. 2014 Korean liver cancer study group-national cancer center Korea practice guideline for the management of hepatocellular carcinoma. Korean J Radiol. 2015;16:465-522.

13. Omata M, Cheng AL, Kokudo N, et al. Asia-Pacific clinical practice guidelines on the management of hepatocellular carcinoma: a 2017 update. Hepatol Int. 2017;11:317-370.

14. Ye J-Z, Wang -Y-Y, Bai T, et al. Surgical resection for hepatocellular carcinoma with portal vein tumor thrombus in the Asia-Pacific region beyond the barcelona clinic liver cancer treatment algorithms: a review and update. Oncotarget. 2017;8:9325-9378.

15. Agarwal PD, Phillips P, Hillman L, et al. Multidisciplinary management of hepatocellular carcinoma improves access to therapy and patient survival. J Clin Gastroenterol. 2017;51:845-849. doi:10. 1097/MCG.0000000000000825

16. Liu P-H, Lee Y-H, Hsia C-Y, et al. Surgical resection versus transarterial chemoembolization for hepatocellular carcinoma with portal vein tumor thrombosis: a propensity score analysis. Ann Surg Oncol. 2014;21:1825-1833. doi:10.1245/s10434-014-3510-3

17. Zhang XP, Gao YZ, Chen ZH, et al. An EHBH-PVTT scoring system as an aid to decision-making on hepatectomy for HCC patients with PVTT: a multicenter study. Hepatology. 2018. doi:10.1002/hep.30490.
18. Huo L, Wei W-X, Yan Z-L, et al. Short-term and long-term outcomes of liver resection for HCC patients with portal vein tumor thrombus. Cell Biosci. 2019;9:23. doi:10.1186/s13027-019-0229-6.

19. Navadgi S, Chang CC, Bartlett A, McCall J, Pandanaboyana S. Systematic review and meta-analysis of outcomes after liver resection in patients with hepatocellular carcinoma (HCC) with and without bile duct thrombus. HPB. 2016;18:312-316.

20. Oba A, Takahashi S, Kato Y, et al. Usefulness of resection for hepatocellular carcinoma with macroscopic bile duct tumor thrombus. Anticancer Res. 2014;34:4367-4372.

21. Wang C, Yang Y, Sun D, Jiang Y. Prognosis of hepatocellular carcinoma patients with bile duct tumor thrombus after hepatic resection or liver transplantation in Asian populations: A meta-analysis. PLoS One. 2017;12:e0176827. doi:10.1371/journal.pone.0176827

22. Yu SJ, Kim YJ. Effective treatment strategies other than sorafenib for the patients with advanced hepatocellular carcinoma invading portal vein. World J Hepatol. 2015;7:1553-1561. doi:10.4254/wjh.v7. i11.1553

23. Liver cancer study group of Japan. General rules for the clinical and pathological study of primary liver cancer, Second English edition edn. Kanehara \& Co, Tokyo, 2003; pp 34-35.

24. Shuqun C, Mengchao W, Han C, et al. Tumor thrombus types influence the prognosis of hepatocellular carcinoma with the tumor thrombi in the portal vein. Hepatogastroenterology. 2007;74:499-502.

25. Ueda M, Takeuchi T, Takayasu T, et al. Classification and surgical treatment of hepatocellular carcinoma (HCC) with bile duct thrombi. Hepatogastroenterology. 1994;41:349-354.

26. Bruix J, Qin S, Merle P, et al. Regorafenib for patients with hepatocellular carcinoma who progressed on sorafenib treatment (RESORCE): a randomised, double-blind, placebo-controlled, phase 3 trial. Lancet. 2017;389:56-66. doi:10.1016/S0140-6736(16)32453-9

27. Abou-Alfa GK, Meyer T, Cheng AL, et al. Cabozantinib in patients with advanced and progressing hepatocellular carcinoma. $N$ Engl $J$ Med. 2018;379:54-63. doi:10.1056/NEJMoa1717002

28. Zhu AX, Park JO, Ryoo BY, et al. Ramucirumab versus placebo as second-line treatment in patients with advanced hepatocellular carcinoma following first-line therapy with sorafenib (REACH): a randomised, double-blind, multicentre, phase 3 trial. Lancet Oncol. 2015;16:859-870. doi:10.1016/S1470-2045(15)00050-9

29. Kokudo T, Hasegawa K, Matsuyama Y, et al. Survival benefit of liver resection for hepatocellular carcinoma associated with portal vein invasion. J Hepatol. 2016;65:938-943. doi:10.1016/j.jhep.2016.05. 044

30. Yoon SM, Ryoo BY, Lee SJ, et al. Efficacy and safety of transarterial chemoembolization plus external beam radiotherapy vs sorafenib in hepatocellular carcinoma with macroscopic vascular invasion: a randomized clinical trial. JAMA Oncol. 2018;4:661-669. doi:10.1001/jamaoncol.2017.5847

31. Meng K, Dong M, Zhang W, Huang Q. Clinical characteristics and surgical prognosis of hepatocellular carcinoma with bile duct invasion. Gastroenterol Res Pract. 2014;604971.

32. Xiuping Z, Juxian S, Kang W, Shuqun C. The current status of diagnosis and treatment of hepatocellular carcinoma with cholangiocarcinoma. Chin J Hepat Surg. 2017;5:343. doi:10.3877/ cma.j.issn.2095-3232.2017.05.002

33. Wu Z, Guo K, Sun H, et al. Caution for diagnosis and surgical treatment of recurrent cholangitis: lessons from 5 cases of bile duct tumor thrombus without a detectable intrahepatic tumor. Medicine. 2014;93(11):e80 . . doi:10.1097/MD.0000000000000080

34. Xiangji L, Weifeng T, Bin Y, et al. Surgery of hepatocellular carcinoma complicated with cancer thrombi in bile duct: efficacy for criteria for different therapy modalities. Langenbecks Arch Surg. 2009;394:1033-1039. doi:10.1007/s00423-009-0496-6

35. Yu XH, Xu LB, Liu C, et al. Clinicopathological characteristics of 20 cases of hepatocellular carcinoma with bile duct tumor thrombi. Dig Dis Sci. 2011;56:252-259. doi:10.1007/s10620-010-1256-8 
36. Satoh S, Ikai I, Honda G, et al. Clinicopathologic evaluation of hepatocellular carcinoma with bile duct thrombi. Surgery. 2000;128 (5):779-783. doi:10.1067/msy.2000.108659

37. Chotirosniramit A, Liwattanakun A, Lapisatepun W, Ko- Iam W, Sandhu T, Junrungsee S. A single institution report of 19 hepatocellular carcinoma patients with bile duct tumor thrombus. $J$ Hepatocell Carcinoma. 2017;4:41. doi:10.2147/JHC.S126308
38. Zeng H, Xu L, Wen J, et al. Hepatocellular carcinoma with bile duct tumor thrombus. Medicine. 2015;94(1):e364.

\section{Publish your work in this journal}

Cancer Management and Research is an international, peer-reviewed open access journal focusing on cancer research and the optimal use of preventative and integrated treatment interventions to achieve improved outcomes, enhanced survival and quality of life for the cancer patient.
The manuscript management system is completely online and includes a very quick and fair peer-review system, which is all easy to use. Visit http://www.dovepress.com/testimonials.php to read real quotes from published authors. 